from 5.3\% (CI95: 4.2-6.7) in men aged $18-29$ to $1.8 \%$ (CI95: 1.0-3.3) in men aged 70 and over. However, at the same time, the proportion of falls among all UIs rises with age: from $22.7 \%$ (CI95: 18.4-27.7) to 52.6\% (CI95: 32.7-71.7) in men and from 20.1\% (CI95:15.2-26.0) to 63.9\% (CI95: 47.7-77.5) in women. The proportion of falls that occur at home also increases significantly with age: from $14 \%$ to over 50\%. Still, among the elderly, about one third of falls occurs in public places (among pedestrians). The consequences of UIs caused by falls are often serious: 34.4\% (CI95:29.8-39.4) of all falls cause bone fractures (all UIs: 20.4\%; CI95: 18.3-22.6). One-quarter (26.8\%; CI95: 22.431.7) result in hospitalisation (all UIs: 18.3\%; CI95: 16.2-20.5). Moreover, the need for physical therapy and rehabilitation is higher after falling compared to all other UIs.

Conclusions The data demonstrate the severity of UIs caused by falls and reveal important age and gender specific patterns. Older people are no more at risk than younger people, but the consequences for them are more serious than for the younger. Consequently, fall-related UIs are of importance in all ages and require age and gender specific approaches to prevention.

\section{DEVELOPING A MODEL FOR PROCESSING THE INFORMATION OF ROAD TRAFFIC INJURIES AS THE DATABASE FOR COLLABORATIVELY PLANNING OPERATIONAL MEASURES TO REDUCE THE ROAD TRAFFIC ACCIDENTS}

'Patcharawadee Pirunsuntorn, ${ }^{2}$ Wilai Thanalad, ${ }^{1}$ Adisak Khongwattananont. ${ }^{1}$ Khonkaen Provincial Public Health Office; ${ }^{2}$ Kranuan Crown Prince Hospital

\subsection{6/injuryprev-2016-042156.733}

Background Based on the global status report on road safety in 2013 by World Health Organisation (WHO), Thailand ranked third in the list of countries having highest road traffic deaths worldwide (reported number of road traffic deaths Estimated number of road traffic deaths; Point estimate 26,312 Estimated road traffic death rate per 100000 population approximately 26,312 or at the death rate of 38 road fatalities per 100,000 inhabitants). The median value of the total number of road fatality in Khonkaen during2011-2013 was the fourth highest nationwide (468 or at the death rate of 26.49 road fatalities per 100,000 inhabitants).

Description of the problem The information from the injury surveillance and the dead case investigation recorded by Khonkaen provincial public health office have not been utilised at its maximum efficiency; less information can be accessible by the network units. Consequently, each unit has individually managed its assignment in solving the problems on road traffic accidents. Thus, to integrate the operational procedures of all the units concerned in order to create a co-operation and collaborative operational guidelines for them to follow, Khonkean provincial public health office arranged a venue for returning the information to those units in parallel with developing a model in processing the recorded information of road traffic injuries as the database available for all the network units to easily access into the system and make use of the records for collaboratively planning the operational measures serving the safety road scheme.

Results Obtained a model for managing the information processing systemby integrating the operational procedures of all the network units beginning from the regular surveillance, the case investigation, to the delivery of information to the communities and encouraged to have the findings utilised for integrated problem solving both in short term and long term; and 2) establishment of community checkpoints at the village level to reduce road traffic accidents during festival seasons.

Conclusions Obviously, social tendency of realisation alert for safety road was widely enhanced; this was the result from the understanding of the road traffic safety and the integration of problem solving procedures for road traffic accidents among all the parties concerned.

\section{FACTORS INFLUENCING SOCIAL AND HEALTH OUTCOMES AFTER LAND TRANSPORT INJURY: RECRUITMENT AND PARTICIPANT CHARACTERISTICS, SHORT TERM HEALTH AND SOCIAL STATUS}

Jagnoor Jagnoor, Ian Cameron, on behalf of the FISH study investigators. University of Sydney, Australia

\subsection{6/injuryprev-2016-042156.734}

Background There is growing evidence that health and social outcomes following motor vehicle crash injury are related to cognitive and emotional responses of the injured individual, as well as relationships between the injured individual and the compensation systems with which they interact. Investigation is therefore warranted to identify the key determinants of health and social outcomes following injury in the context of the New South Wales motor accident insurance scheme.

Methods In this inception cohort study, 2400 participants, aged 17 years or more, injured in a motor vehicle crash in New South Wales are being identified principally though hospital emergency departments. Participants will be initially contacted through mail. Baseline interviews are conducted by telephone within 28 days of the injury and participants are followed up with at 6, 12 and 24 months post-injury. Medicare and Pharmaceutical prescription data will also be linked to the observed data.

Results Recruitment for the study is underway with 1438, 924, 706, 135 baseline, 6, 12 and 24 months interviews completed respectively. Interim analysis of first 777 participants is reported below. Over one- fourth $(215 ; 27.3 \%)$ of the participants were born outside Australia, 67\% were males, $79.5 \%$ were in paid employment at the time of injury. The data source/hospital was significantly associated with the distribution of mode of transport injuries and major differences were observed for urban hospitals with $35.9 \%(232 / 647)$ of the cohort being bicyclists whilst a high proportion of motorcycle riding injuries $(51.6 \%$; 48/93) were reported from rural hospitals. At the first interview, participants had a lower health related quality of life than the general population (EQ5D a mean difference of -0.539 ; <0.0001); despite less than half reporting admission to hospital because of their injury. Return to work was reported by $65 \%$ whilst only $36 \%$ reported being able to return to their usual social activities. Analysis of outcome predictors related to post-injury function, disability and return-to-work are now under way.

Conclusions The interim analysis of the cohort reported a very high proportion of bicyclists injuries. Identification of factors associated with health and social outcomes following injury, including related compensation factors will provide evidence for improved service delivery, post-injury management, and inform policy development and reforms. 